\title{
Intractable seizures in a toddler after application of an over-the-counter local anesthetic cream
}

\author{
Syed Hoda MD, Jennifer O'Brien PhD, Jonathan Gamble MD
}

Competing interests: None declared.

This article has been peer reviewed.

The authors have obtained patient consent.

Correspondence to: Jonathan Gamble, j_gamble@yahoo.com

CMAJ 2016. DOI:10.1503 /cmaj.150387

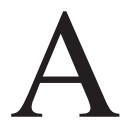
17-month-old boy was brought to the emergency department for dressing changes to first- and second-degree burns. He had been burned on the chin, neck and chest from scalding liquid at home the previous day. The burns affected about $15 \%$ of his body surface area. The patient weighed $10 \mathrm{~kg}$. His vaccinations were up to date, and he had been previously well.

The patient's pain was being controlled with oral morphine and codeine. Before bringing him to the emergency department, his parents had spontaneously purchased Polysporin Kids Cream, which contains 5\% lidocaine hydrochloride by weight. They requested that the cream be applied during the dressing change. The health care team applied the entire $15-\mathrm{g}$ tube of the cream, which contained $750 \mathrm{mg}$ of lidocaine in total, during the dressing change.

About 20 minutes later, while waiting for discharge, the child had a generalized tonic-clonic seizure. His airway was secured with an endotracheal tube, without sedation because there was no intravenous access in place. Intraosseous access was established, and four doses of both lorazepam (total $4 \mathrm{mg}$ ) and midazolam (total $4 \mathrm{mg}$ ) were administered via this access; however, the seizures continued. After establishment of intravenous access, propofol and fentanyl were administered, followed by phenobarbital, which subsequently controlled the seizures.

Following cessation of the seizures, a loading

\section{KEY POINTS}

- Although topical anesthetics used appropriately are safe, inappropriate use can result in potentially life-threatening outcomes.

- Application of topical anesthetics over burned skin increases systemic absorption.

- Children may not complain of common preconvulsive signs and symptoms associated with toxicity from a topical anesthetic, such as lightheadness, dizziness, and visual and auditory disturbances.

- When toxicity from a topical anesthetic are suspected, serum levels of the anesthetic and its metabolites should be measured. dose of phenytoin was administered. Midazolam and fentanyl infusions were started for maintenance sedation. A dopamine infusion was begun because of hypotension and titrated to keep the patient's systolic blood pressure above $80 \mathrm{~mm} \mathrm{Hg}$. Acyclovir, cefotaxime and vancomycin were given intravenously to treat possible infectious causes of the seizures.

Initial arterial blood gas levels were as follows: $\mathrm{pH} 7.2$, partial pressure of carbon dioxide $\left(\mathrm{PaCO}_{2}\right) 47 \mathrm{~mm} \mathrm{Hg}$, partial pressure of oxygen $119 \mathrm{~mm} \mathrm{Hg}$, bicarbonate $18 \mathrm{mmol} / \mathrm{L}$, base excess -10 and oxygen saturation $97.8 \%$. The fraction of inspired oxygen was $60 \%$, and the methemoglobin level was $1.1 \%$. Blood samples obtained about two and five hours after initial lidocaine exposure were sent to the provincial laboratory for lidocaine measurement. The first sample was not processed, and the second showed a serum lidocaine level of less than $1 \mu \mathrm{mol} / \mathrm{L}$. Cerebral spinal fluid analysis showed clear fluid, with normal values for cell count, glucose, protein and lactate dehydrogenase levels. An initial toxic screen was positive only for opiates. A computed tomography scan showed no obvious pathology.

The patient was transferred to an out-ofprovince pediatric intensive care unit after his condition was stabilized. He was extubated the next day, weaned from inotropes and transferred back to his local hospital. The intravenous treatment with acyclovir, cefotaxime and vancomycin was continued until subsequent cultures showed no bacterial or viral infection, and the patient was discharged home. No other sources of toxins or fever were identified on further history-taking, and the patient and his family had no history of seizures.

At follow-up two weeks after discharge, the patient had had no further seizures and had normal findings on an electroencephalogram. In the approximately three and a half years since then, the patient has remained seizure free. The case was reported to Vigilance Canada. 


\section{Discussion}

The cause of the patient's seizure was likely lidocaine toxicity originating from topical application of the lidocaine cream ("probable/likely" classification according to the World Health Organization-Uppsala Monitoring Centre causality assessment criteria: www.who-umc.org/Graphics/ 24734.pdf).

Although early post-exposure lidocaine levels were not obtained, the seizures were attributed to the lidocaine cream based on its known pharmacodynamics and time from application to onset of seizure. In addition, the patient's medical history was negative for seizures, he had had no further seizures at follow-up, and investigations for other causes of seizures, including lumbar puncture, blood chemistry, neuroimaging, electroencephalography and blood culture, did not support an alternative diagnosis.

\section{Establishing a probable diagnosis of lidocaine toxicity}

The lidocaine in Polysporin Kids Cream is a solid pure base and forms an oil-in-water emulsion, which allows a higher concentration of the active ingredient per emulsion droplet for enhanced skin penetration. ${ }^{1}$ Manufacturer's warnings prohibit use over large areas of the body, in deep or puncture wounds, on animal bites or on serious burns (www.polysporin.ca/products/for-kids). The amount of lidocaine administered in our case was $750 \mathrm{mg}(75 \mathrm{mg} / \mathrm{kg})$ on burned skin over $15 \%$ of the patient's body surface area. The recommended maximum dose of topical lidocaine is $4.5 \mathrm{mg} / \mathrm{kg} .{ }^{2}$ Based on the patient's weight, the administered dose was 15 times the recommended intravenous dose.

There is little in the literature to guide dosing of topical anesthetics over burn sites, other than the absorption appears higher when the product is applied over thermal injury than over surgically induced wounds. ${ }^{3}$ The burn dressings may have also increased the risk of toxicity in our case. Removal of the dressings and remaining lidocaine cream was not considered during the acute resuscitation period because the cause of the patient's seizures had not yet been attributed to lidocaine toxicity.

The association of seizures with lidocaine is well established. ${ }^{4}$ Our case differs because of the route of administration, although we found one case of systemic toxicity associated with mucosal application of lidocaine. ${ }^{5}$

Serum levels of lidocaine and its metabolites would have confirmed the diagnosis of lidocaine toxicity in our case. Blood levels were drawn two and five hours after the seizure, but the first sample was not processed and the second was taken too late to detect toxic lidocaine levels. The half-life of lidocaine in children is about one hour; its metabolites monoethylglycine xylidide and glycine xylidide stay in plasma much longer, with terminal half-lives of 2 and 10 hours, respectively. ${ }^{6}$ Measurement of the serum metabolite levels was not ordered in our case.

Our patient was reported to have gone from a sleep state to convulsions, which is different from the usual progression of preconvulsive symptoms. Toxicity symptoms in awake adults develop at lidocaine levels of $4.5-7.5 \mu \mathrm{g} / \mathrm{mL}$. Increasing levels of local anesthetic correlate with a progression of symptoms, including circumoral paresthesia, lightheadedness, restlessness, confusion, dizziness, visual and auditory disturbances, and tinnitus. The signs of central nervous system (CNS) toxicity are a progression of shivering, slurred speech, and muscle twitching and tremors initially involving the face and distal extremities. $^{7}$ The preconvulsive symptoms of our patient may have been short-lived and missed by his caregivers because serum lidocaine levels rose rapidly. When the plasma concentration of lidocaine increases, CNS excitation occurs, followed by tonic-clonic generalized convulsions, as witnessed in our patient. ${ }^{7}$

\section{Pathophysiology of lidocaine toxicity}

The mechanism of initial CNS excitation and seizures is attributed to the preferential blockade of inhibitory cortical synapses and unopposed excitatory activity by lidocaine and its active metabolite, monoethylglycine xylidide. ${ }^{4}$ This excitation is thought to result from the blockade of inhibitory pathways; it may also result from the net increase of glutamate, an excitatory amino acid neurotransmitter. ${ }^{8}$ Further increase in the dose of lidocaine leads to inhibition of activity of both the inhibitory and facilitatory circuits, which results in a generalized state of CNS depression. ${ }^{8}$ In our patient, CNS depression from lidocaine toxicity did not evolve after CNS excitation; instead, it occurred after active management of the seizures with sedatives and anticonvulsants.

In addition to generalized CNS depression secondary to a sufficiently large dose or rapid injection of lidocaine, respiratory depression or arrest can occur. ${ }^{7}$ Our patient was intubated because of obtundation before respiratory arrest occurred. Early control of the airway and ventilatory support is critical.

Because seizures contribute to a combined respiratory and metabolic acidosis, rapid correction of hypercapnia and acidosis reduces further CNS toxicity. Increased $\mathrm{PaCO}_{2}$ levels enhance cerebral blood flow, thus delivering lidocaine 
more rapidly to the brain. ${ }^{8}$ In addition, diffusion of carbon dioxide into neuronal cells decreases the intracellular $\mathrm{pH}$ level, which facilitates conversion of the base form of the drug to the cationic form. This diminishes the outward diffusion of lidocaine across the nerve membrane via ion trapping, which further increases CNS toxicity. Hypercapnia and acidosis decrease the plasma protein binding of lidocaine agents. Plasma protein binding is the most important pharmacologic factor that determines the toxicity of lidocaine, because it is the free fraction of the drug that produces toxicity. Of note, plasma concentrations of $\alpha_{1}$-acid glycoprotein are lower in neonates than in adults and older children, and thus it is recommended that lidocaine doses be reduced by about $30 \%$ in neonates and infants. ${ }^{7}$

In adults, cardiovascular toxicity usually follows CNS toxicity. The hemodynamic changes are a result of dilation of the peripheral vasculature and direct myocardial depression, with progressive bradycardia and cardiac arrest. ${ }^{8}$ A dopamine infusion was needed in our patient, probably because of a combination of cardiovascular effects from lidocaine toxicity and adverse effects from administration of sedatives and anticonvulsants.

Other issues with lidocaine toxicity include methemoglobinemia, although this was not a concern in our patient. Methemoglobinemia is much less likely to be associated with lidocaine exposure than with prilocaine or benzocaine toxicity. ${ }^{9}$

\section{Preventing similar incidents}

The parents' suggestion to apply the lidocaine cream was understandable, because topical anesthetics have been shown to be effective in reducing pain in many superficial procedures, including dressing changes. ${ }^{10-12}$ Further, the product information for Polysporin Kids Cream indicates that it is "suited for minor cuts, scrapes and burns" (www.polysporin.ca/products/for-kids).

The narrow view of the cause of this case's events was that the maximum safe dose of topical lidocaine was exceeded. Factors that allowed this overdose to occur are less clear but may include the product's easy availability over the counter, a lack of clarity or misinterpretation of the product information, and a lack of knowledge by the health care team of the total dose of lidocaine applied and the product's increased absorption on burned skin.

To prevent this type of occurrence, application of topical lidocaine should not exceed maximum dosage recommendations, with the type of wound taken into consideration. Solutions to address this type of error must be multifactorial and include both individual education and systemic precautions. This may include limiting the sale of this and similar products to behind-the- counter dispensing and updating product instructions to include weight-based dosing guidelines.

We recognize that weight-based dosing alone may be difficult for the lay public to interpret; hence, we recommend following other over-thecounter medication models that include both weight- and age-based dosing instructions. The latter would enable pharmacists to provide safety advice about the product to patients and caregivers.

Although emergency department staff are experienced in using topical anesthetics, many may lack experience in their use over burns. In addition, when caregivers bring in outside medications, a routine safety check by the hospital pharmacy is eliminated. Preventing the use of medications brought by caregivers for administration in the hospital or mandatory pharmacy consultation for use of such medications may enhance patient safety. Finally, reporting such events to a central postmarket surveillance program such as Vigilance Canada (www.hc-sc.gc.ca/dhp-mps/medeff/ vigilance-eng.php) is important.

\section{References}

1. Burkhart C, Morrell DK, Goldsmith L. Dermatological pharmacology. In: Brunton LL, Chabner BA, Knollmann BC, editors. Goodman \& Gilman's the pharmacological basis of therapeutics. 12th ed. McGraw-Hill; 2011:1803-32.

2. Lexicomp Online: Pediatric \& Neonatal Lexi-Drugs Online [database]. Hudson (OH): Lexi-Comp; 2015. Sept. 22, 2015.

3. Read JM, Bach PH. Sterile topical lignocaine jelly in plastic surgery: an assessment of its systemic toxicity. S Afr Med J 1980; 57:704-6.

4. Catterall WA, Mackie K. Local anesthetics. In: Brunton LL, Chabner BA, Knollmann BC, editors. Goodman \& Gilman's the pharmacological basis of therapeutics. 12th ed. McGraw-Hill; 2011:565-82.

5. Mofenson HC, Caraccio TR, Miller H, et al. Lidocaine toxicity from topical mucosal application. Clin Pediatr (Phila) 1983;22: 190-2.

6. Oni G, Brown S, Burrus C, et al. Effect of $4 \%$ topical lidocaine applied to the face on the serum levels of lidocaine and its metabolite, monoethylglycinexylidide. Aesthet Surg J 2010;30:853-8.

7. Polaner DM, Suresh S, Coté CJ. Regional anesthesia. In: Coté CJ, Lerman J, Todres ID, editors. A practice of anesthesia for infants and children. 4th ed. Philadelphia: Saunders; 2009:869-70.

8. Berde CB, Strichartz GR. Local anesthetics. In: Miller RD, editor. Miller's anesthesia. 8th ed. Seattle: Saunders; 2014:1028-54.

9. Guay J. Methemoglobinemia related to local anesthetics: a summary of 242 episodes. Anesth Analg 2009;108:837-45.

10. Chen BK, Cunningham BB. Topical anesthetics in children: agents and techniques that equally comfort patients, parents, and clinicians. Curr Opin Pediatr 2001;13:324-30.

11. Sobanko JF, Miller CJ, Alster TS. Topical anesthetics for dermatologic procedures: a review. Dermatol Surg 2012;38:709-21.

12. Desai C, Wood FM, Schug SA, et al. Effectiveness of a topical local anaesthetic spray as analgesia for dressing changes: a doubleblinded randomised pilot trial comparing an emulsion with an aqueous lidocaine formulation. Burns 2014;40:106-12.

Affiliation: Department of Anesthesiology, Perioperative Medicine and Pain Management, University of Saskatchewan, Saskatoon, Sask.

Contributors: Jennifer O'Brien and Jonathan Gamble contributed to the conception and design of the manuscript. Syed Hoda drafted the manuscript, and all of the authors revised it for important intellectual content. All of the authors approved the final version to be published and agreed to act as guarantors of the work. 